\title{
Using a Dictionary and $n$-gram Alignment to Improve Fine-grained Cross-Language Plagiarism Detection
}

\author{
Nava Ehsan* \\ School of Electrical and \\ Computer Engineering \\ College of Engineering \\ University of Tehran \\ Tehran, Iran \\ n.ehsan@ece.ut.ac.ir
}

\author{
Frank Wm. Tompa \\ David R. Cheriton School of \\ Computer Science \\ University of Waterloo \\ Waterloo, ON, Canada \\ N2L 3G1 \\ fwtompa@uwaterloo.ca
}

\author{
Azadeh Shakery \\ School of Electrical and \\ Computer Engineering \\ College of Engineering \\ University of Tehran \\ Tehran, Iran \\ shakery@ut.ac.ir
}

\begin{abstract}
The Web offers fast and easy access to a wide range of documents in various languages, and translation and editing tools provide the means to create derivative documents fairly easily. This leads to the need to develop effective tools for detecting cross-language plagiarism. Given a suspicious document, cross-language plagiarism detection comprises two main subtasks: retrieving documents that are candidate sources for that document and analyzing those candidates one by one to determine their similarity to the suspicious document. In this paper we focus on the second subtask and introduce a novel approach for assessing cross-language similarity between texts for detecting plagiarized cases. Our proposed approach has two main steps: a vector-based retrieval framework that focuses on high recall, followed by a more precise similarity analysis based on dynamic text alignment. Experiments show that our method outperforms the methods of the best results in PAN-2012 and PAN-2014 in terms of plagdet score. We also show that aligning $n$-gram units, instead of aligning complete sentences, improves the accuracy of detecting plagiarism.
\end{abstract}

\section{CCS Concepts}

-Information systems $\rightarrow$ Near-duplicate and plagiarism detection; Dictionaries; Multilingual and cross-lingual retrieval; Digital libraries and archives; $\bullet$ Applied computing $\rightarrow$ Language translation; Document analysis;

\section{Keywords}

Cross-language plagiarism detection; $n$-grams; bilingual dictionaries; text alignment

\section{INTRODUCTION}

Plagiarism refers to unauthorized use of text, code, music, images, video and ideas [1]. With the rapid growth of documents in

\footnotetext{
${ }^{*}$ Visiting Scholar, University of Waterloo, 2015-16
}

Permission to make digital or hard copies of all or part of this work for personal or classroom use is granted without fee provided that copies are not made or distributed for profit or commercial advantage and that copies bear this notice and the full citation on the first page. Copyrights for components of this work owned by others than ACM must be honored. Abstracting with credit is permitted. To copy otherwise, or republish, to post on servers or to redistribute to lists, requires prior specific permission and/or a fee. Request permissions from permissions@ acm.org.

DocEng '16, September 12-16, 2016, Vienna, Austria

(C) 2016 ACM. ISBN 978-1-4503-4438-8/16/09 . \$ $\$ 15.00$

DOI: http://dx.doi.org/10.1145/2960811.2960817 various languages, the increased accessibility of electronic documents, the availability of translation tools, and the simplicity of cutand-paste, cross-language plagiarism has become a serious problem, and its detection requires more attention. In automatic crosslanguage plagiarism detection, the task is to identify and isolate plagiarized text passages within suspicious documents, where each such passage has originated from a source document written in a language other than the one used in the suspicious document. A prototypical example of document plagiarism is depicted in Figure 1 , where multiple source documents are used, plagiarized passages are of varying size, and plagiarized text from a source can be re-ordered. In cross-language plagiarism, passages from the source documents are also translated into the language of the suspicious document.

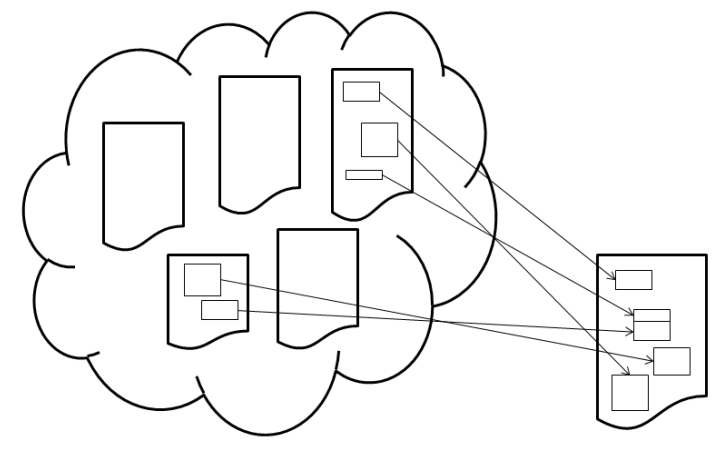

Source Documents

Suspicious Document

Figure 1: Prototypical plagiarism

Given a suspicious document, cross-language plagiarism detection comprises two main tasks: candidate retrieval and pairwise assessment of document similarity [23, 2]. Given a document suspected to include plagiarism, the goal of candidate retrieval is to identify source documents that might contain passages that match fragments of the suspicious document [11]. Assessing pairwise document similarity requires detailed comparison of the suspicious document with each candidate source document in turn to identify the plagiarized fragments precisely. In this paper we focus on this second subtask: given a candidate source document $d$ in language $L$ and a suspicious document $d^{\prime}$ in language $L^{\prime}$, determine which fragments of the suspicious document are plagiarized from fragments in the source document.

Most previous methods for isolating plagiarized fragments are based on translating one of the two documents into the language 
of the other and then applying monolingual techniques [2]. We present an alternative approach that has two steps: a vector-based retrieval framework that focuses on high recall, followed by a more precise similarity measurement based on dynamic text alignment. In the vector-based framework, instead of translating the entire document, we translate selected words and phrases only. For this simpler translation task, lexical knowledge of translation can be taken from any language translation resource. In the second step, only the suspicious fragments identified in the first step are fully translated with a machine translator; these are then aligned to the source document and tested for similarity.

We evaluate the performance in terms of precision, recall, and granularity scores as well as the plagdet score, which is a combination of the first three measures, as defined by Potthast et al. [26]. These evaluation metrics are described in Section 4.1. Our method achieves a higher plagdet score than the methods with best result in PAN-2012 ${ }^{1}$ [24] and in PAN-2014 ${ }^{2}$ [27]. We show that aligning $n$-grams, non-overlapping runs of $n$ words for a small value of $n$, instead of aligning complete sentences, improves the accuracy of detecting plagiarism.

The rest of the paper is organized as follows. In Section 2, we review related work. Section 3 describes our two-step approach, followed in Section 4 by a description of the experimental framework and the presentation of our results. Finally, we summarize our conclusions and outline some future work in Section 5.

\section{RELATED WORK}

Plagiarism detection can use style analysis to detect parts of the text that are inconsistent in terms of writing style (intrinsic meth$o d s$, or it can match suspicious passages in a text to the source(s) for those passages (so-called external methods) [1]. Plagiarism research has primarily addressed the monolingual problem, for which the sources are in the same language as the suspicious text. Monolingual external methods have been based on fingerprint indexing [28, 29], string matching [8], using sequences of stopwords [30], vector-based models [15], probabilistic models [3], semantic-based approaches [7], or classification [22].

In cross-language plagiarism detection, the suspicious document or potential source document(s) can be translated into the language of the other document(s) and a monolingual approach can then be applied. Alternatively, a cross-language similarity method can be used [23, 12], in which texts are compared based on extracted features such as individual words (and their translations) or $n$-grams.

Cross-Language Character $N$-Gram (CL-CNG) [20] is a syntaxbased method that uses overlapping character $n$-grams of the texts for comparing multilingual documents. This approach is applicable for detecting plagiarism among documents written in languages with similar syntax, but it is ineffective when the languages differ syntactically.

CL-ASA [4], LSI [10] and KCCA [31] use parallel corpora in order to find cross-language similarity. Cross-Language Explicit Semantic Analysis (CL-ESA) [25], which is the cross-lingual generalization of ESA [14], uses comparable corpora for this purpose. Alternative approaches to detect word and phrase translations can be based on multi-lingual dictionaries or thesauri [6].

Barrón-Cedeño et al. [2] compared the performance of a syntaxbased approach (CL-CNG), a parallel-corpus-based approach (CLASA), and an approach using translation followed by monolingual analysis (T+MA). In their experiments, conducted on the special

\footnotetext{
${ }^{1}$ http://www.uni-weimar.de/medien/webis/events/pan-12/pan12web/plagiarism-detection.html

${ }^{2}$ http://www.gelbukh.com/plagiarism-detection/PAN-2014/
}

case where the suspicious document is an exact copy of a source document, CL-CNG and T+MA outperform CL-ASA. A drawback of full machine translation, however, is that it is computationally expensive. Furthermore, there is a lack of good automatic translators for some language pairs [9]. Franco-Salvador et al. [12, 13] show that their knowledge graph analysis (CL-KGA) using BabelNet, a multi-lingual semantic network, also outperforms the CLCNG and CL-ASA methods in detecting plagiarism. They further showed that weighing both the concepts and the relations between the concepts in their knowledge graph, using distributed representation of concepts outperforms the CL-ESA method [13]. We show that our approach outperforms the T+MA approaches presented in PAN-2012 [17] and PAN-2014 [27]. We also show that using a dictionary outperforms using BabelNet in our approach.

\section{TWO-STEP RETRIEVAL MODEL}

Given a source document $d$ written in language $L$ and a suspicious document $d^{\prime}$ in language $L^{\prime}$, let $o_{1}, \ldots, o_{n}$ represent the offsets of the $n$ characters in $d$ and let $o_{1}^{\prime}, \ldots, o_{m}^{\prime}$ represent the offsets of the $m$ characters in $d^{\prime}$. Let a fragment of a document be represented by a subset of that document's offsets, where the offsets within a fragment are all contiguous. (For simplicity, we use the same notation $d$ to represent a document or its set of offsets.) Our proposed method aims to detect all fragments of the suspicious document, $d_{f^{\prime}}^{\prime} \subseteq d^{\prime}$, that have been plagiarized from source fragments $d_{f} \subseteq d$. This is depicted in Figure 2, in which four fragments have been plagiarized. Thus, if the first fragment in document $d$ covers offsets $o_{i}, \ldots, o_{j}$ and the corresponding fragment in document $d^{\prime}$ covers offsets $o_{p}^{\prime}, \ldots, o_{q}^{\prime}$, our problem is to detect that $\left\{\left[d, o_{i}\right], \ldots,\left[d, o_{j}\right]\right\} \cup\left\{\left[d^{\prime}, o_{p}^{\prime}\right], \ldots,\left[d^{\prime}, o_{q}^{\prime}\right]\right\}$ is an instance of plagiarism.

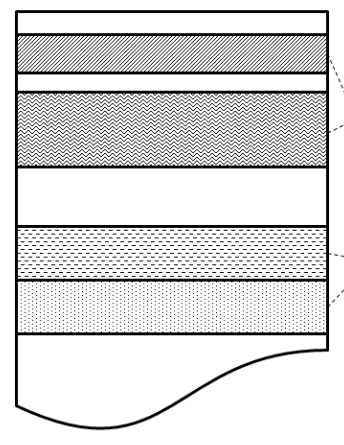

Source Document, $d$

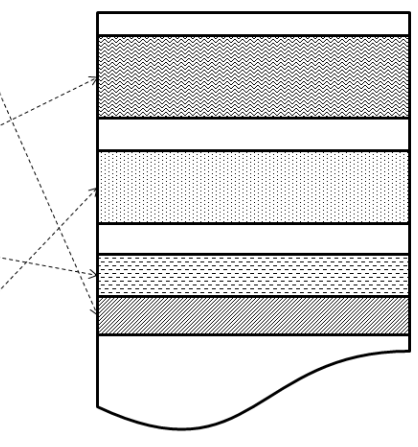

Suspicious Document, $d^{\prime}$
Figure 2: Fine-grained plagiarism in a pair of documents

Thus, when comparing two documents for plagiarism, we need to find a matching of segments from the source text with segments from the suspicious one. In a brute-force approach, such a matching can be defined by first partitioning each text into segments and then pairing the segments from each text. If there are $N$ words in the source text and $M$ words in the target text, there are $2^{N-1}$ and $2^{M-1}$ possible partitions, respectively, having $O(N)$ and $O(M)$ segments respectively. The number of possible matchings is thus $O\left(2^{(N+M)} N M\right)$. Instead of using brute force, the first step in our method tries to find many possibly plagiarized fragments. The aim of the second step is to filter the results by finding alignments between the identified passages and measuring the similarity between aligned fragments more thoroughly. In light of this second step 


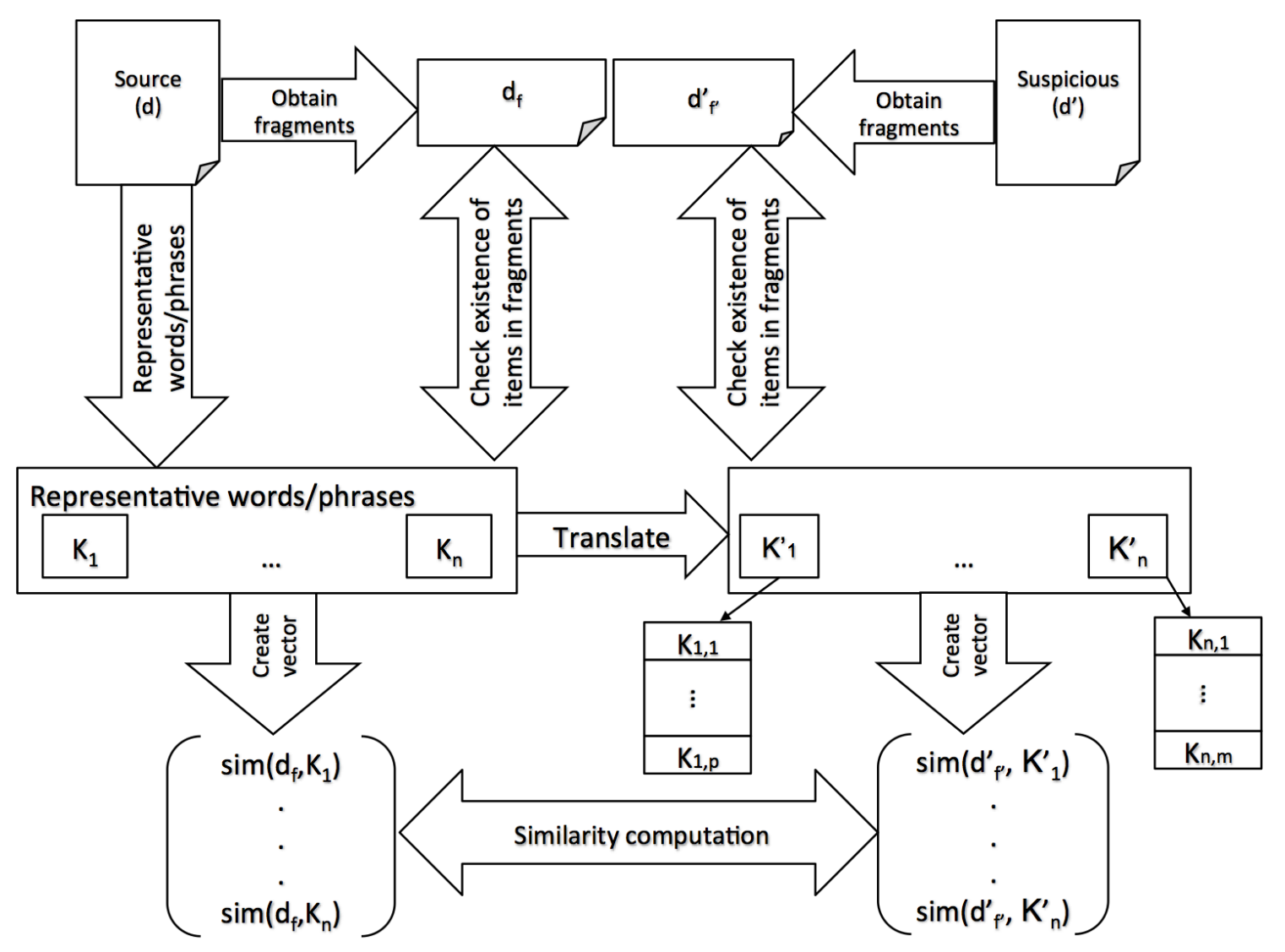

Figure 3: Step 1: Identifying potentially plagiarized fragments

that emphasizes precision, the first step is designed to favour high recall.

\subsection{Candidate sentence identification: high re- call step}

Given a document pair in different languages, we want to identify any plagiarized parts. Rather than translating every word, however, we observe that a sentence in the suspicious document may be a translation of a sentence in the source document if it contains many words that are translations of representative terms from the source sentence. We start by identifying terms in the source document that are indicative of its choice of vocabulary. We then isolate fragments of the source document and of the suspicious document, identify which representative terms appear in each source fragment, and identify which translations of representative terms appear in each suspicious fragment. Finally we pair source and suspicious fragments when they are sufficiently similar to each other, based on the number of representative words and their translation they have in common. This process is depicted in Figure 3 and described in more detail in the remainder of this section.

\subsubsection{Extraction of representative words and phrases}

We use two common statistics adopted from information retrieval to select representative terms: term frequency $(t f)$ and inverse document frequency (idf) [19]. A term's frequency reflects the prominence of that term in a document's vocabulary. This property is even more pronounced for multi-word phrases than for individual words because many complex or technical concepts and product names are expressed with multi-word compounds. Furthermore, small sequences of words that appear often in a document serve as excellent signatures when identifying plagiarized text.

To build our vocabulary of representative terms, we preprocess a source document by removing punctuation, extra white space, and digits, by converting the text to lowercase, and normalizing diacritic characters; this yields a sequence of consistently generated word tokens. We next include all unigrams (single word tokens, not including stopwords) that have a frequency above half of the maximum frequency of any term in the document. We next include those bigrams (pairs of consecutive word tokens) having a frequency above half of the maximum frequency of any bigram in the document, and all trigrams (triples of consecutive word tokens) appearing more than twice in the document. (We allow extracted bigrams and trigrams to contain at most one stopword each.) Inverse document frequency reflects the selectivity in distinguishing a document's vocabulary from the vocabulary of other documents. Therefore, we also include those unigrams of a document that appear in no other document in the collection.

Finally, we identify additional representative unigrams by adapting a technique from query expansion. More specifically, we use a mixture model for pseudo-relevance feedback [32], available in the Lemur toolkit ${ }^{3}$, assuming that each document is relevant to itself. We then include the top 20 such unigrams.

\subsubsection{Candidate sentence selection}

Having identified a set of representative terms that appear in the source document, we produce a vector space $K_{1}, \ldots, K_{n}$ in the language of the source document and a corresponding vector

\footnotetext{
${ }^{3}$ http://www.lemurproject.org/
} 
space of those terms' translations $\mathcal{K}_{1}^{\prime}, \ldots, \mathcal{K}_{n}^{\prime}$ in the language of the suspicious document. We use a set of translations $\left(\mathcal{K}_{i}^{\prime}\right)$ for each term $\left(K_{i}\right)$ instead of relying on one translation only, so that we include paraphrased translations and improve recall. Notably, lexical knowledge for translation can be taken from dictionaries, semantic networks, or any other external language resource.

The next problem is choosing which fragments to test as the smallest detectable unit of plagiarism. In general any sequence of words in the source text might match any sequence of words in the suspicious text. If the texts have $N$ and $M$ words, respectively, this requires testing $O\left(N^{2} M^{2}\right)$ pairs and considering translations for each of the $O\left(N^{2}\right)$ sequences. Instead, we split the documents into sentences, recording the position and length of each sentence. We assume that examples of plagiarism involve at least $k$ consecutive sentences, with tuning parameter $k$. We therefore isolate $k$ sentence fragments from each document, using a sliding window over the sentences of the document. As a result, each fragment is created from a sequence of $k$ consecutive sentences, and two fragments from a document may overlap by as many as $k-1$ sentences. This is depicted in Figure 4. As a result, we need to test only $O(N M)$ fragment pairs, and we compare only some chosen terms rather than using full translations.

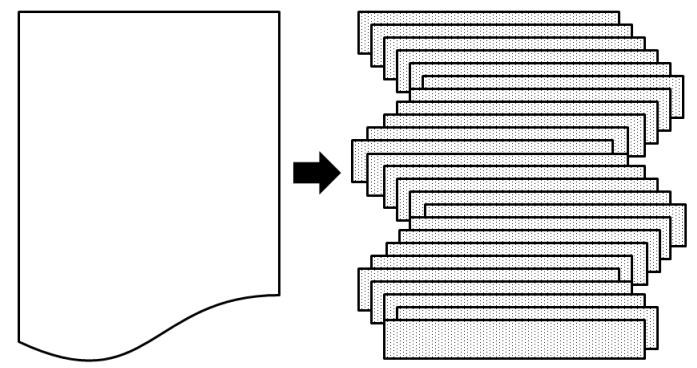

Figure 4: Overlapping $k$-sentence fragments produced by sliding window

For each fragment $d_{f}$ in the source document, we create a vector in the space of representative terms by setting $v_{d_{f}}\left[K_{i}\right]$ to 1 if the term $K_{i}$ occurs in the fragment and 0 otherwise. Similarly, if any of the translations $K^{\prime} \in \mathcal{K}_{i}^{\prime}$ of $K_{i}$ appears in a fragment identified by $d_{f^{\prime}}^{\prime}$ in the suspicious document, we set $v_{d_{f^{\prime}}^{\prime}}\left[K_{i}^{\prime}\right]$ to 1 . Although the precision decreases by allowing any of the translations, the second step of our approach, described in the next section, has as its goal to improve precision.

Next the representative term vectors for each fragment in the source document are compared to the term vectors for fragments in the suspicious document. $d_{f}$ will be considered as a potential plagiarism source of $d_{f^{\prime}}^{\prime}$ if its representative term vector has maximum similarity to that of $d_{f^{\prime}}^{\prime}$ among all term vectors for fragments in document $d$ and that similarity is above a threshold obtained from a training set. Before passing the pairs of potential plagiarism instances to the second step of the algorithm, adjacent pairs are merged if they fall within a fixed proximity threshold in both the source and suspicious documents.

Figure 5 shows one matched pair of fragments consisting of five sentences and eight sentences, respectively, the latter resulting from merging three overlapping windows. The highlighted words in each fragment are the representative words and phrases that indicate that this might be a plagiarized piece of text, with the lighter highlighting indicating those words that match in the other text. Because representative terms in the source document are the only elements being translated, it is not crucial to have high quality ma-

\section{Source fragment:}

Die kontinuierliche Überwachung der legislativen Arbeit durch die Wähler, das Parlament und die Massenmedien kann durch eine gemeinsame Datenbank erleichtert werden, die den Namen des Verantwortlichen in der Kommission und des Berichterstatters des Europäischen Parlaments enthält .

Ferner sollte sie Angaben zum Zeitplan, zu den Auswirkungen auf den Haushalt sowie zur Rechtsgrundlage umfassen.

Diese Datenbank sollte öffentlich zugänglich sein und wäre meiner Meinung nach auch für unsere Kollegen in den einzelstaatlichen Parlamenten von großem Nutzen.

Herr Präsident, ich hoffe, die Kommissionsmitglieder werden bald, vielleicht schon in den nächsten Tagen, Kontakt mit den Ausschüssen des Parlaments aufnehmen, damit schnellstmöglich mit der Umsetzung des vereinbarten Zeitplans und der festgelegten Arbeitsformen begonnen werden kann.

Ich glaube, dass mit einer derartigen Änderung der Verfahren die Arbeitsweise und der Legislativzyklus effizienter, transparenter und verständlicher gestaltet werden können.

\section{Suspicious fragment:}

To make it easier for the electorate, ourselves and the mass media to follow the legislative work, a common database should be set up showing who in the Commission and Parliament is responsible for a particular proposal.

The database should also contain information about the calendar, budgetary implications and legal base

The database should be publicly available.

I believe it should prove to be of very great help to our colleagues in the national parliaments.

Mr President, I hope that, no later than in the course of the next few days, the Commissioners will begin to visit Parliaments' committees so that a start can be made as soon as possible on following the agreed timetable and method of working .

About three o'clock in the afternoon during the warm weather Mother would begin skimming the milk, carrying it pan by pan to the big cream pan, where with a quick movement of a case knife the cream was separated from the sides of the pan, the pan tilted on the edge of the cream pan and the heavy mantle of cream, in folds or flakes, slid off into the receptacle and the thick milk emptied into pails to be carried to the swill barrel for the hogs.

I used to help Mother at times by handing her the pans of milk from the rack and emptying the pails.

Then came the washing of the pans at the trough, at which I also often aided her by standing the pans up to dry and sun on the big bench.

Figure 5: A matched pair of fragments detected in the first step, with representative terms highlighted

chine translation between the two languages for this step. There is no need to translate the entire text, and therefore this step of our approach is applicable to languages that have any translation re- 
source. In particular, it is applicable for low-resourced languages but improves as more translations are available for consideration.

\subsection{Result filtering: focusing on precision}

In the previous step, we retrieved potentially plagiarized, multisentence fragments signalled by their use of representative terms. In this step, we translate the suspicious fragments only, using a full machine translation system, and then we filter out those instances that have poor similarity when all words other than stopwords are considered. By filtering out false positive detections, the hope is that this step improves the precision, and hence the overall performance, of the model.

A plagiarized fragment may omit pieces from the source, but it is likely that at least some of the smallest units of discourse are preserved in their original order. Therefore, for this step of the algorithm, we partition the sentences within a fragment into non-overlapping $n$-grams (i.e., sub-sentence units consisting of $n$ consecutive words, on average ${ }^{4}$ ) and try to align such segments in the source fragment with corresponding segments in the suspicious fragment to which it has been paired in the first step. This stage of alignment aims at pairing corresponding segments without reordering ${ }^{5}$, and it accommodates situations in which a plagiarized text might merge or split segments in the translated document. This is illustrated in Figure 6, where the first segment in the source fragment does not appear in the suspicious one, the second segment is split into two segments in the suspicious fragment, the third and fourth segments are merged into a single segment, and the fifth segment is translated as a single segment in the suspicious document.

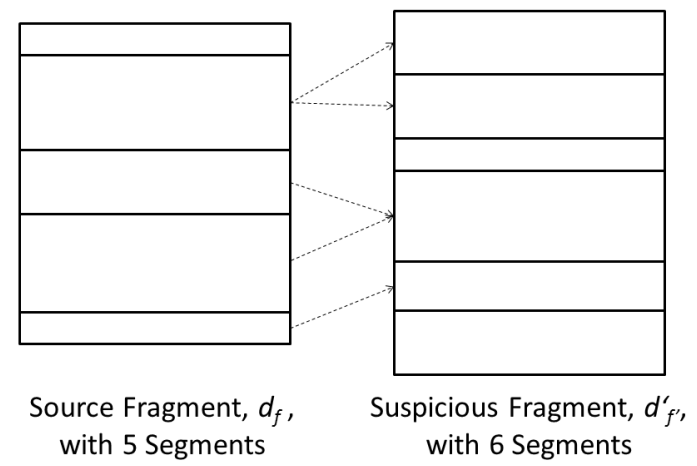

Figure 6: Aligning segments within a pair of fragments

The alignment score is obtained by a dynamic programming algorithm based on the one proposed by Ma [18] and using a measure of string similarity sim:

$$
S(i, j)=\max \left\{\begin{array}{l}
S(i-1, j) \\
S(i, j-1) \\
S(i-1, j-1)+\operatorname{sim}\left(\operatorname{susp}_{i}, \operatorname{src}_{j}\right) \\
S(i-1, j-2)+\operatorname{sim}\left(\operatorname{susp}_{i}, \operatorname{src}_{j-1: j}\right) \\
S(i-1, j-3)+\operatorname{sim}\left(\operatorname{susp}_{i}, \operatorname{src}_{j-2: j}\right) \\
S(i-2, j-1)+\operatorname{sim}\left(\operatorname{susp}_{i-1: i}, \operatorname{src}_{j}\right) \\
S(i-3, j-1)+\operatorname{sim}\left(\text { susp }_{i-2: i}, \text { src }_{j}\right)
\end{array}\right.
$$

For simplicity, we assume that translations will split or merge at most three segments at a time, and we therefore allow 1-0, 0-1, 1-

\footnotetext{
${ }^{4}$ In fact for a sentence of length $s$, the number of partitions is chosen to be $s / n$ rounded to the nearest integer, and the final partition may include fewer or more than $n$ terms.

${ }^{5}$ Text rearrangements are accommodated in the first step of the algorithm, described in Section 3.1.
}

1, 1-2, 2-1, 1-3 and 3-1 alignments. The optimal alignment of the first $i$ suspicious segments and $j$ source segments depends on the alignment score of some sequential prefix of these segments plus a single alignment. $S(i, j)$ represents the similarity from the beginning of the documents to the $i^{\text {th }}$ suspicious segment and the $j^{\text {th }}$ source segment. The notation $s u s p_{x: y}$ represents the concatenation of suspicious segments numbered from $x$ to $y$, and similarly $s r c_{x: y}$ refers to source segments. We modified the algorithm proposed by Ma [18], to implicitly penalize 1-0 and 0-1 alignments and also to make all scores comparable. To this end, we keep track of the number of alignments obtained so far, and the score in each step is normalized by the number of the alignments. Thus, null alignments (i.e., 1-0 and 0-1 alignments) are penalized because they only increase the count of the alignments but not the similarity score. If the optimal alignment score is above a threshold, that case is reported as a plagiarized case. Finally, complete sentences at the start or end of a fragment having more than half their content in a 1-0 or 01 alignment are excluded from the reported suspicious and source cases, respectively.

Figure 7 shows the results of applying the dynamic program to the pair of matched fragments shown in Figure 5. The final pruning step drops the last two sentences of the suspicious text, but keeps all sentences from the source text because more than half of the first and last sentences contribute to the alignment.

\section{EXPERIMENTAL FRAMEWORK}

Our experiments examine the step-by-step performance of the proposed approach and serve several purposes: (1) evaluating the candidate sentence selection algorithm using three distinct external resources for translation (Section 4.2), (2) evaluating the dynamic alignment algorithm and comparing various levels of granularity for alignment purpose (Section 4.3), and (3) evaluating the effectiveness of determining pairwise document similarity by applying the proposed two step retrieval model (also in Section 4.3).

\subsection{Data sets and evaluation metrics}

We evaluate our proposed approach using the complete GermanEnglish partition of the PAN-PC-12 text alignment corpus ${ }^{6}$. Both the training data set and the test data set include plagiarized documents that are obfuscated by translation from German into English. The training set comprises 305 source and 179 suspicious documents, among which some suspicious documents contain plagiarized text from more than one source document and some source documents are plagiarized in more than one suspicious document, yielding 449 pairs. In the test set there are 263 pairs for which each suspicious document has exactly one corresponding source document. The construction principles of the corpus are defined by Potthast et al. [26], who also define the following evaluation metrics.

Let $S$ and $R$ represent the set of true plagiarism cases and the reported detections, respectively, where $s \in S$ (and similarly $r \in R$ ) is the union of the sets of offsets in a plagiarized fragment from the suspicious document and in the corresponding fragments from the source document (each offset marked with the document to which it refers). Given source document $d$ and suspicious document $d^{\prime}$, we say that $r \in R$ detects $s \in S$, written as $\delta(r, s)$, if $r \cap s$ includes at least one offset from each document:

$$
\delta(r, s) \Leftrightarrow(r \cap s \cap d \neq \emptyset) \wedge\left(r \cap s \cap d^{\prime} \neq \emptyset\right)
$$

The performance of the task is reported as the macro-average plagdet score, which is a combination of the macro-average precision $\mathcal{P}$,

\footnotetext{
${ }^{6}$ http://www.uni-weimar.de/medien/webis/events/pan-12/pan12web/plagiarism-detection.html
} 
Source fragment (translated):

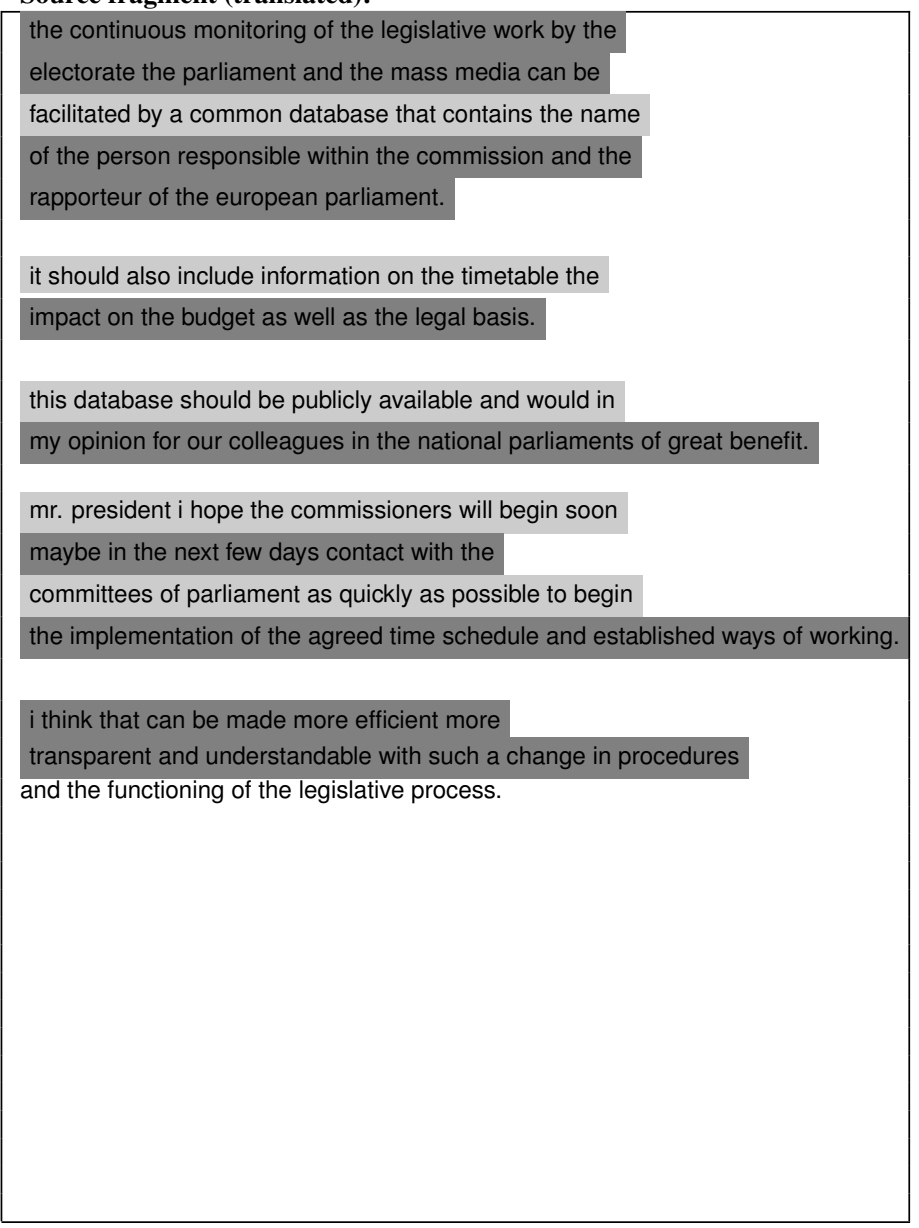

Suspicious fragment:

to make it easier for the electorate ourselves and
the mass media to follow the legislative work a
common database should be set up showing who in
the commission and parliament is responsible for a particular proposal.
the database should also contain information about the calendar
budgetary implications and legal base.
the database should be publicly available.
i believe it should prove to be of very
great help to our colleagues in the national parliaments.
mr president i hope that no later than in
the course of the next few days the commissioners
will begin to visit parliaments' committees so that a
start can be made as soon as possible on
following the agreed timetable and method of working.
about three o'clock in the afternoon during the warm
weather mother would begin skimming the milk carrying it
pan by pan to the big cream pan where
with a quick movement of a case knife the
cream was separated from the sides of the pan
the pan tilted on the edge of the cream
pan and the heavy mantle of cream in folds
or flakes slid off into the receptacle and the
thick milk emptied into pails to be carried to
the swill barrel for the hogs.
i used to help mother at times by handing
her the pans of milk from the rack and emptying the pails.
then came the washing of the pans at the
trough at which i also often aided her by
standing the pans up to dry and sun on the big bench.

Figure 7: Aligned 9-grams in the matched fragments from Fig. 5

recall $\mathcal{R}$, and granularity $\mathcal{G}$ metrics defined as follows:

$$
\begin{gathered}
\mathcal{P}(R, S)=\frac{1}{|R|} \sum_{r \in R} \frac{\left|\bigcup_{s \in S}(s \sqcap r)\right|}{|r|} \\
\mathcal{R}(R, S)=\frac{1}{|S|} \sum_{s \in S} \frac{\left|\bigcup_{r \in R}(s \sqcap r)\right|}{|s|} \\
\mathcal{F}_{1}(R, S)=\frac{2 \mathcal{P}(R, S) \mathcal{R}(R, S)}{\mathcal{P}(R, S)+\mathcal{R}(R, S)} \\
\mathcal{G}(R, S)=\frac{1}{\left|S_{R}\right|} \sum_{s \in S_{R}}\left|R_{s}\right|
\end{gathered}
$$

where $s \sqcap r=s \cap r$ if $\delta(r, s)$ and $\emptyset$ otherwise, $S_{R}=\{s \in$ $S \mid \exists r \in R: \delta(r, s)\}$, and $R_{s}=\{r \in R \mid \delta(r, s)\}$. Precision, recall, and $F_{1}$ (their harmonic mean) are adapted from information retrieval. Granularity is a metric specific to plagiarism detection that reports the average number of reported instances of plagiarism per detected source fragment of plagiarism. These scores are combined into an overall score called plagdet and defined as follows ${ }^{7}$ :

$$
\operatorname{plagdet}(R, S)=\frac{\mathcal{F}_{1}(R, S)}{\log _{2}(1+\mathcal{G}(\mathcal{R}, \mathcal{S}))}
$$

\footnotetext{
${ }^{7}$ This measure is used in PAN's plagiarism detection competition.
}

\subsection{Evaluating the performance of candidate sentence identification}

In the first step of the algorithm we use the framework described in Section 3.1. We use the training data to tune two parameters. The first one is the number of consecutive sentences to include in each fragment, which represents the smallest detectable unit of plagiarism. The second parameter is the threshold for accepting a pair of fragments as evidence of plagiarism.

Our approach limits candidate fragments to align with sentence boundaries and include at least $k$ sentences. Based on experiments using the training data, we set $k=5$. (Coincidentally, 5-sentence sliding windows are also used by Franco-Salvador et al. as the fragment size for plagiarism detection [13].) Recall that each fragment from the source and suspicious document is represented as a binary vector $\left\langle\operatorname{sim}_{1}, \operatorname{sim}_{2}, \ldots, \operatorname{sim}_{n}\right\rangle \in\{0,1\}^{n}$ as described in Section 3.1. We identify source passage $d_{f}$ and suspicious passage $d_{f^{\prime}}^{\prime}$ as a plagiarism candidate pair if the source passage term vector has maximum similarity to that of $d_{f^{\prime}}^{\prime}$ among all term vectors for fragments in document $d$ and their cosine similarity exceeds threshold $t$, which is set to 0.4 based on the training data. In order to eliminate spurious matches, we also require that the source fragment includes translations of at least three keywords found in the suspicious fragment.

To improve performance with respect to the granularity metric, 


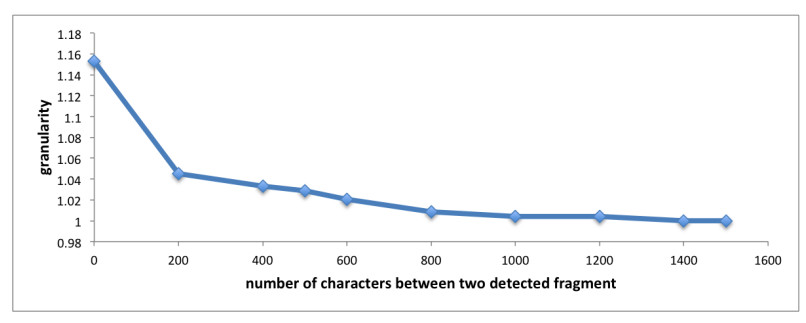

Figure 8: Effect of merging adjacent fragments on the granularity metric

which distinguishes whether an instance of plagiarism is detected as a whole or in several pieces, we merge two adjacent detected fragments to report a single plagiarism case if the number of characters between those fragments in the source and suspicious documents are both below a fixed proximity threshold ${ }^{8}$. Because this threshold is not obtained from training, we measure the sensitivity of the proximity parameter and display the results in Figure 8. Granularity starts at 1.1529 without merging adjacent fragments and decreases to 1 as more characters are allowed to exist between two adjacent fragments. The proximity threshold of 1500 chosen for our experiments falls well within the range that produces a granularity score of 1 . The resulting passages are candidate result pairs consisting of five or more sentences each, which are further processed in the next step.

We test three distinct external resources for the translation phase: Google translate ${ }^{9}$, BabelNet $^{10}$, and dict.cc ${ }^{11}$. Google offers a machine translation service, from which we used the best translation provided to serve as a baseline. BabelNet is a multi-lingual semantic network, in which each node represents a concept and the edges record the semantic relationships between the concepts; concepts and relations are selected from WordNet and Wikipedia [21]. The dict.cc dictionary is a German-English dictionary containing $1,070,884$ entries.

Without applying a German stemmer and without splitting compound words, only $28 \%$ of the representative terms appear in BabelNet and $43 \%$ appear in dict.cc. We therefore apply a lightweight preprocessing step to improve the coverage: we use the jwordsplitter toolkit ${ }^{12}$ to split German compounds and a German stemmer based on the algorithm described by Caumanns [5]. We stemmed words from the document and also from dict.cc.

The process of looking up a term is then as follows. We look up each term (unigram, bigram, or trigram) in the original reference work. If a unigram is not found, the stem of the term is looked up (but in the stemmed dictionary for dict.cc). If the term includes multiple words after splitting compounds, each word is treated individually. If all parts of such a term are found in either the original or stemmed reference work, all their translations are recorded. Otherwise we assume that the term is not translated, and the original term is kept as the translation of the term (in case the term is a proper name that is repeated identically in the other language). After applying this preprocessing step, the average percentage of terms found in dict.cc increases to about $81 \%$ and in BabelNet increases to about $48 \%$.

\footnotetext{
${ }^{8} \mathrm{We}$ could just as well have chosen to measure proximity in terms of numbers of words.

${ }^{9}$ https://translate.google.ca/

${ }^{10} \mathrm{http} / / /$ babelfy.org/

${ }^{11} \mathrm{http}: / /$ www.dict.cc/

${ }^{12}$ https://github.com/danielnaber/jwordsplitter
}

Table 1 shows that the approach using Google translation has the highest plagdet score at the end of the first step, but the highest recall is achieved by using the dict.cc dictionary with preprocessing. By keeping more translations for each word, we are able to catch paraphrased translations and translations using alternative language resources. The exceedingly low precision of this step and the correspondingly poor plagdet score result from not considering the positions of the words, the presence of many possible translations, and the coincidental similarity resulting from the use of common words. In the next step, however, we will eliminate many false positive detections to improve precision.

\subsection{Evaluating the two-step approach}

The second set of experiments focuses on the filtering step. Since we are looking for text fragments borrowed from another source, the objective is to avoid reporting documents with coincidental similarity between texts.

For this step, we apply the Google translation system to the potentially plagiarized source fragments only. Figure 9 is a stacked graph showing for each document the number of sentences, the number of sentences we translate, and the number of plagiarized sentences. The amount of translation required in our experiments is about $45 \%$ of the sentences from the test data, in which approximately $33 \%$ of the entire test data sentences are plagiarized. Thus we have significantly reduced the use of an expensive component, and this reduction increases if the suspicious documents include fewer or smaller plagiarized segments.

Those fragments containing randomly common words do not signal plagiarism, and the dynamic alignment algorithm takes into account the position and proximity of the common words. To identify an appropriate threshold to distinguish between true positive and false positive plagiarism detection, we use a subset of our training data, namely 112 cases retrieved from the first step of the algorithm together with their translations. The average similarity scores calculated by applying the second step of the algorithm on this training set is chosen as the threshold.

Table 2 shows the results of the detailed analysis experiment using the dynamic alignment algorithm and how it behaves with both sentence-level and $n$-gram-level granularity for segments, where $n$ is set to 9 based on runs conducted with the training data. We can see that using $n$-grams outperforms using sentences by about $4.4 \%$, which is statistically significant (Wilcoxon signed rank test $p<0.0001$ ), and running the dynamic algorithm with more items to align was only slightly slower than running the algorithm with sentences instead. The overall score is also compared with the methods of the best results in PAN-2012 [17] and PAN-2014 [27], both of which use monolingual techniques for which the translations of the entire documents of the source collection are used. For this experiment, we applied the algorithms described in the earlier papers to the same test collection used for our approach (obtaining slightly different performance results than reported in the original papers on other corpora).

For completeness, we also tested our approach using segments that correspond to elementary discourse units (EDUs), which are clause-like fragments that serve as building blocks of sentences [16]. We hypothesized that since EDUs are semantically coherent lexical units rather than arbitrary sequences of $n$ consecutive words of text, they might be better representatives of the atomic units of plagiarism. EDUs were extracted using the CODRA toolkit ${ }^{13}$, requiring 402 minutes to execute for all the candidate fragments identified in the first step of our approach (whereas partitioning into equiva-

\footnotetext{
${ }^{13}$ https://www.cs.ubc.ca/cs-research/lci/research-groups/naturallanguage-processing/Discourse.html
} 
Table 1: Results of using various translation resources for the first step of the algorithm

\begin{tabular}{|c|cccc|}
\hline External Language Resource & Precision & Recall & Granularity & Plagdet \\
\hline Google translate & $\mathbf{0 . 7 2 6 7}$ & 0.7839 & $\mathbf{1}$ & $\mathbf{0 . 7 5 4 2}$ \\
BabelNet (without preprocessing) & 0.7067 & 0.3487 & $\mathbf{1}$ & 0.4670 \\
BabelNet (with preprocessing) & 0.5337 & 0.6379 & $\mathbf{1}$ & 0.5811 \\
Dict.cc (without preprocessing) & 0.5733 & 0.6377 & $\mathbf{1}$ & 0.6038 \\
Dict.cc (with preprocessing) & 0.2960 & $\mathbf{0 . 8 7 2 1}$ & $\mathbf{1}$ & 0.4419 \\
\hline
\end{tabular}

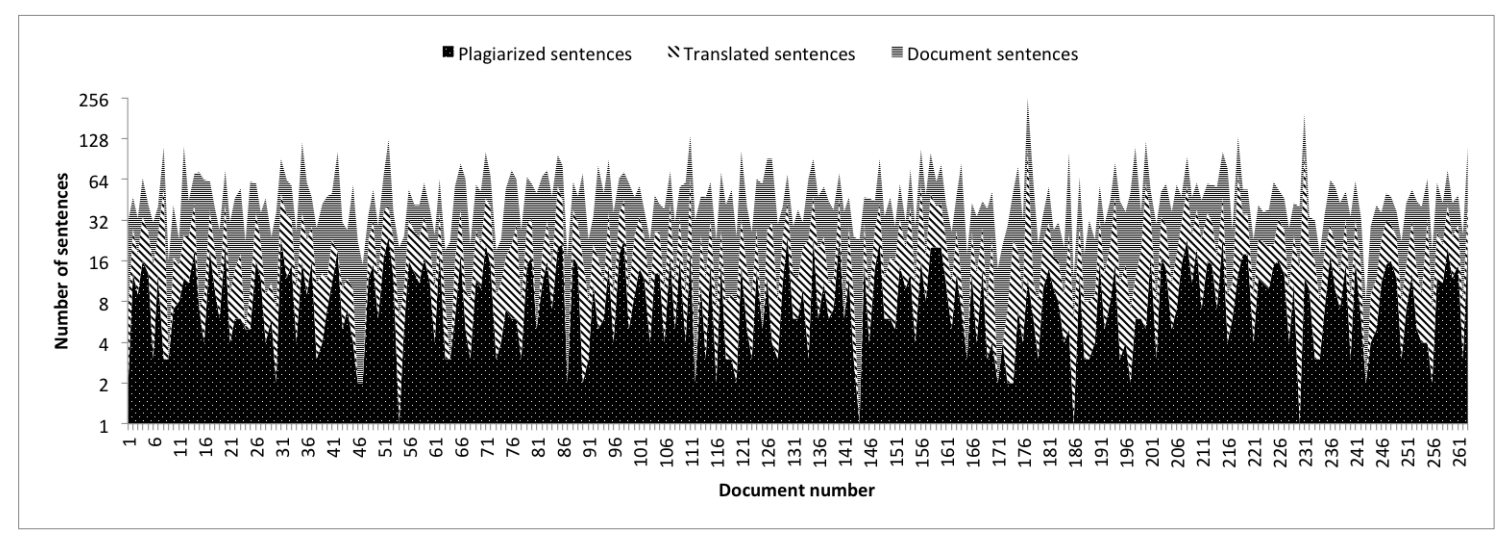

Figure 9: Amount of translation required compared to the number of sentences and the amount of plagiarism

Table 2: Comparing the results of pairwise document similarity assessment

\begin{tabular}{|c|cccc|}
\hline Method & Precision & Recall & Granularity & Plagdet \\
\hline Best result from PAN-2012 & 0.8040 & 0.6976 & $\mathbf{1}$ & 0.7470 \\
Best result from PAN-2014 & 0.8125 & 0.7205 & $\mathbf{1}$ & 0.7637 \\
Using dict.cc and sentence granularity & 0.8405 & $\mathbf{0 . 8 2 9 3}$ & $\mathbf{1}$ & 0.8348 \\
Using Google translate and $n$-gram granularity & 0.9214 & 0.7505 & $\mathbf{1}$ & 0.8272 \\
Using dict.cc and $n$-gram granularity & $\mathbf{0 . 9 3 0 1}$ & 0.8193 & $\mathbf{1}$ & $\mathbf{0 . 8 7 1 2}$ \\
Using dict.cc and EDU granularity & 0.9190 & 0.8204 & $\mathbf{1}$ & $\mathbf{0 . 8 6 6 9}$ \\
\hline
\end{tabular}


lently sized $n$-grams is essentially instantaneous). Our experimentally chosen value of $n=9$ for $n$-gram splitting is, perhaps not coincidentally, fairly close to the average EDU size, which is about 7 for the training data and about 8.6 for the test data. As it turns out, the difference in performance when using EDUs as compared to $n$-grams is not statistically significant.

To complete our comparison, Table 2 also includes the results from applying our second step to the fragments identified by Google translate in the first step; as expected, the lower recall in the first step results in a lower performance overall. Thus we conclude that using dict.cc in the first step and using $n$-gram granularity in the second step results in best performance.

\section{CONCLUSION AND FUTURE WORK}

In this paper we propose a two-step retrieval model for detecting cross-language plagiarized sentences given the source and suspicious documents. The first step selects candidate sentences, with a goal of high recall, and the second step aims to filter out false positive detections with an alignment model connecting similar segments. Lexical similarity cues used in the vector representation of the first step identifies similar fragments which could signal plagiarism. This accommodates rearrangements of plagiarized fragments as well as restricting the dynamic programming algorithm of the next step to highly suspicious regions.

The impact of three distinct translation resources (Google, BabelNet and dict.cc) are evaluated in the first step of the algorithm. The experimental results show that using multiple senses from a dictionary, together with some simple lexical compound-splitting and stemming, outperforms the other resources with respect to recall for retrieving potential similarities between sentences of the two languages.

To avoid reporting coincidental similarities as plagiarism, the fragments retrieved in the first step are further processed with a more precise measure using dynamic text alignment. The alignment algorithm is tested with different levels of granularity: sentences, EDUs, and $n$-grams. Experiments show that using EDUs slightly outperforms using sentence level alignment, but requires significantly more execution time. However, splitting a text into $n$ gram units performs as well as using EDUs, but with less execution time. The proposed approach outperforms today's best translation and monolingual techniques and cuts translation time significantly.

For further work, we would like to devise a method to apply the same framework without requiring any sentence translation in the second step. Furthermore, our approach should be combined with a high-quality candidate identification mechanism [11] to produce an effective end-to-end system for cross-language plagiarism detection.

\subsection{Acknowledgments}

The use of computing facilities at the University of Tehran and at the University of Waterloo is gratefully acknowledged. We also thank Miguel Sánchez-Pérez et al. [27] for making their code available.

\section{REFERENCES}

[1] A. Barrón-Cedeño. On the mono-and cross-language detection of text reuse and plagiarism. In Proceedings of the 33 rd International ACM SIGIR Conference on Research and Development in Information Retrieval, pages 914-914. ACM, 2010.

[2] A. Barrón-Cedeño, P. Gupta, and P. Rosso. Methods for cross-language plagiarism detection. Knowledge-Based Systems, 45(1):45-62, 2013.
[3] A. Barrón-Cedeño, P. Rosso, and J.-M. Benedí. Reducing the plagiarism detection search space on the basis of the Kullback-Leibler distance. In Computational Linguistics and Intelligent Text Processing, pages 523-534. Springer, 2009.

[4] A. Barrón-Cedeño, P. Rosso, D. Pinto, and A. Juan. On cross-lingual plagiarism analysis using a statistical model. In Working Notes for CLEF 2008 Conference; PAN Evaluation Lab: Uncovering Plagiarism, Authorship and Social Software Misuse, pages 9-13, 2008.

[5] J. Caumanns. A fast and simple stemming algorithm for German words. Technical report, Free University of Berlin, 1999.

[6] Z. Ceska, M. Toman, and K. Jezek. Multilingual plagiarism detection. In Proceedings of the 13th International Conference on Artificial Intelligence: Methodologies, Systtems, and Applications (AIMSA 2008), pages 83-92, 2008.

[7] C.-Y. Chen, J.-Y. Yeh, and H.-R. Ke. Plagiarism detection using Rouge and WordNet. Journal of Computing, pages 34 $-44,2010$.

[8] P. Clough and M. Stevenson. Developing a corpus of plagiarised short answers. Language Resources and Evaluation, 45(1):5-24, 2011.

[9] V. Danilova. Cross-language plagiarism detection methods. In the Student Research Workshop associated with Recent Advances in Natural Language Processing, RANLP, pages 51-57, 2013.

[10] S. T. Dumais, T. A. Letsche, M. L. Littman, and T. K. Landauer. Automatic cross-language retrieval using latent semantic indexing. In AAAI Spring Symposium on Cross-language Text and Speech Retrieval, volume 15, pages 18-24, 1997.

[11] N. Ehsan and A. Shakery. Candidate document retrieval for cross-lingual plagiarism detection using two level proximity information. Information Processing \& Management, 2016.

[12] M. Franco-Salvador, P. Gupta, and P. Rosso. Cross-language plagiarism detection using a multilingual semantic network. In Advances in Information Retrieval, pages 710-713. Springer, 2013.

[13] M. Franco-Salvador, P. Rosso, and M. Montes-y Gómez. A systematic study of knowledge graph analysis for cross-language plagiarism detection. Information Processing \& Management, 52(4):550-570, 2016.

[14] E. Gabrilovich and S. Markovitch. Computing semantic relatedness using Wikipedia-based explicit semantic analysis. In International Joint Conference on Artificial Intelligence, IJCAI, volume 7, pages 1606-1611, 2007.

[15] C. Grozea, C. Gehl, and M. Popescu. Encoplot: Pairwise sequence matching in linear time applied to plagiarism detection. In Working Notes for CLEF 2009 Conference; PAN Evaluation Lab: Uncovering Plagiarism, Authorship and Social Software Misuse, pages 10 - 18, 2009.

[16] S. Joty, G. Carenini, and R. T. Ng. Codra: A novel discriminative framework for rhetorical analysis. Computational Linguistics, pages 904 - 915, 2015.

[17] K. Leilei, Q. Haoliang, W. Shuai, D. Cuixia, W. Suhong, and H. Yong. Approaches for candidate document retrieval and detailed comparison of plagiarism detection. In Working Notes for CLEF 2012 Conference; PAN Evaluation Lab: Uncovering Plagiarism, Authorship and Social Software Misuse, 2012.

[18] X. Ma. Champollion: A robust parallel text sentence aligner. 
In LREC 2006: Fifth International Conference on Language Resources and Evaluation, pages 489-492, 2006.

[19] C. D. Manning, P. Raghavan, and H. Schütze. Introduction to Information Retrieval, volume 1. Cambridge University Press Cambridge, 2008.

[20] P. McNamee and J. Mayfield. Character $n$-gram tokenization for European language text retrieval. Information Retrieval, 7(1-2):73-97, 2004.

[21] R. Navigli and S. P. Ponzetto. BabelNet: Building a very large multilingual semantic network. In Proceedings of the 48th Annual Meeting of the Association for Computational Linguistics, pages 216-225. Association for Computational Linguistics, 2010.

[22] R. C. Pereira, V. P. Moreira, and R. Galante. A new approach for cross-language plagiarism analysis. In Multilingual and Multimodal Information Access Evaluation, pages 15-26. Springer, 2010.

[23] M. Potthast, A. Barrón-Cedeño, B. Stein, and P. Rosso. Cross-language plagiarism detection. Language Resources and Evaluation, 45(1):45-62, 2011.

[24] M. Potthast, T. Gollub, M. Hagen, J. Kiesel, M. Michel, A. Oberländer, M. Tippmann, A. Barrón-Cedeño, P. Gupta, P. Rosso, et al. Overview of the 4th international competition on plagiarism detection. In Working Notes for CLEF 2012 Conference; PAN Evaluation Lab: Uncovering Plagiarism, Authorship and Social Software Misuse, 2012.

[25] M. Potthast, B. Stein, and M. Anderka. A Wikipedia-based multilingual retrieval model. In Advances in Information Retrieval, pages 522-530. Springer, 2008.
[26] M. Potthast, B. Stein, A. Barrón-Cedeño, and P. Rosso. An evaluation framework for plagiarism detection. In Proceedings of the 23rd International Conference on Computational Linguistics: Posters, pages 997-1005. Association for Computational Linguistics, 2010.

[27] M. A. Sánchez-Pérez, A. F. Gelbukh, and G. Sidorov. Adaptive algorithm for plagiarism detection: The best-performing approach at PAN 2014 text alignment competition. In Proceedings of the 6th International Conference of the CLEF Association, pages 402-413, 2015.

[28] S. Schleimer, D. S. Wilkerson, and A. Aiken. Winnowing: local algorithms for document fingerprinting. In Proceedings of the 2003 ACM SIGMOD International Conference on Management of Data, pages 76-85. ACM, 2003.

[29] J. Seo and W. B. Croft. Local text reuse detection. In Proceedings of the 31st International ACM SIGIR Conference on Research and Development in Information Retrieval, pages 571-578. ACM, 2008.

[30] E. Stamatatos. Plagiarism detection using stopword $n$-grams. Journal of the American Society for Information Science and Technology, 62(12):2512-2527, 2011.

[31] A. Vinokourov, N. Cristianini, and J. S. Shawe-taylor. Inferring a semantic representation of text via cross-language correlation analysis. In Advances in Neural Information Processing Systems, pages 1473-1480, 2002.

[32] C. Zhai and J. Lafferty. Model-based feedback in the language modeling approach to information retrieval. In Proceedings of the Tenth International Conference on Information and Knowledge Management, CIKM '01. ACM, 2001. 